\title{
Evaluation of mechanical properties of unsaturated polyester-guar gum/hydroxypropyl guar gum composites
}

\author{
M. A. Shenoy*, D. J. D’Melo \\ Polymer Engineering and Technology Division, University Institute of Chemical Technology, Nathalal Parikh Marg, \\ Matunga (East), Mumbai, India
}

Received 2 June 2007; accepted in revised form 25 July 2007

\begin{abstract}
Guar gum is a natural polysaccharide that has been explored for various applications. However, there is a limited number of studies in which guar gum has been used as a filler in a polymer. The effect of guar gum and its hydroxypropyl derivatives in unsaturated polyester composites were investigated with respect to their mechanical and chemical properties. The effect of hydroxypropylation and the degree of hydroxypropylation on the properties of resultant composites were also studied. It was observed that the inclusion of guar gum and its derivatives resulted in composites with increased solvent resistance and mechanical properties. An increase in the degree of substitution resulted in increased polymer-filler interaction reflected by a positive effect on the mechanical properties of the composites. These results open an avenue for the use of polysaccharides and their derivatives as eco-friendly fillers as a replacement of mineral fillers.
\end{abstract}

Keywords: polymer composites, mechanical properties, biodegradable polymers, thermosetting resins, material testing

\section{Introduction}

Guar gum is a naturally occurring polysaccharide extracted from the endosperm of Cyamopsis tetragonalobus and structurally is a galactomannan with a galactose to mannose ratio of approximately $1: 2$ [1]. There has been some discussion regarding the distribution of the galactose on the mannose backbone, investigations have indicated that the distribution is not uniform but occurs in blocks with galactose rich and deficient regions $[2,3]$. These galactose deficient regions are not soluble in water while the galactose rich portions are extremely soluble, resulting in the polymer forming a colloidal solution in water. In spite of this, guar gum and its derivatives are extremely water soluble, hydrophilic polymers, the solutions of which are highly viscous in nature. This property has allowed these polymers and their derivatives to be commercialized in fields such as textiles $[4,5]$, foods [6], cos- metics [7], pharmaceuticals [8] and oil recovery and drilling $[9,10]$.

Unsaturated polyester resins are widely used commercial thermosetting resin which are used neat [11], with fillers [12] and reinforced using fibres [13]. Apart from the addition of a separate phase in the polymer matrix, the unsaturated resins have been modified by the use of various reactive diluents [14], reactants [15] and by tailoring the polymer backbone to suit requirements of various applications [16].

The use of guar gum or its derivatives in polymers matrices as fillers has not been investigated in depth. The use of other naturally occurring, renewable polysaccharides as fillers has been focused on starches [17], lignin [18], wood flour [19] and similar materials in mainly thermoplastic polymers. The few studies where guar gum or its derivatives have been used as fillers include the use of guar gum in a polyurethane-polyacrylonitrile interpene-

*Corresponding author, e-mail: mas@udct.org

(c) BME-PT and GTE 
trating network [20] and the incorporation of benzoylated guar gums in a quaternised polysulphone polymer matrix [21]. This study attempts to fill in the lacunae in the information available on the use of guar gum and its derivatives as fillers in an unsaturated polyester matrix.

In this investigation, guar gum and hydroxypropyl guar gums were used as fillers in a conventional unsaturated polyester matrix. The mechanical properties were studied with respect to concentration of fillers added as well as the effect of hydroxy-propylation of the derivatives. Guar gum is obtained from natural sources and is hence a renewable resource, biodegradable and non-toxic. The chemical modification of guar gum, in this case hydroxyl-propylation, is not restricted to the surface of the filler, as in the case treatment of mineral fillers, but occurs throughout the polymer chain and the polysaccharide particle. Thus, the effect of the reaction with guar gum will not only affect the polymer-filler interaction but also change the nature of the filler. We expect any improvement of properties to be solely based on increased physical interaction between filler and polymer and not due to any chemical reaction between the two as is possible in the case of vinyl functionalisation. This paper attempts to investigate the feasibility of using guar gum and hydroxypropyl guar gum as a filler in unsaturated polyester composites, as well as the effect of increasing hydrophobic character of the inherently hydrophilic filler on the properties of the composite.

\section{Experimental}

\subsection{Materials}

The unsaturated polyester resin, cobalt octoate (accelerator) and methyl ethyl ketone peroxide (initiator) was obtained from M/s Mechamco Ltd. India. Guar gum (GG) and hydroxypropyl guar gum with a molar substitution of 0.4 (HPG4) and 0.8 (HPG8) was supplied by M/s Lucid Colloids Ltd., India. The properties of guar gum and deriva-

Table 1. Properties of guar gum and hydroxypropyl guar gum

\begin{tabular}{|l|c|c|c|}
\hline & GG & HPG4 & HPG8 \\
\hline Viscosity (1\% solution) & $1800 \mathrm{cps}$ & $1400 \mathrm{cps}$ & $1200 \mathrm{cps}$ \\
\hline$\%$ moisture & $5-6$ & $6-7$ & $5-6$ \\
\hline Ash content (wt \%) & $0.8-1.0$ & $1.1-1.3$ & $0.9-1.1$ \\
\hline Free propanol (wt \%) & - & $<0.1 \%$ & $<0.1 \%$ \\
\hline
\end{tabular}

tised guar gum are listed in Table 1 and have been obtained from the datasheet provided by M/s Lucid Colloids Ltd., India.

The shape of the guar gum and hydroxypropyl guar gum particles was estimated using an optical microscope, Olympus BX41. The rheology of the unsaturated polyester-guar gum compositions were studied on a RT10 Rheoviscometer using a C $35 / 2^{\circ}$ cone and plate assembly. The readings were measures under constant stress.

\subsection{Preparation of composites}

The fillers were uniformly dispersed in the unsaturated polyester resin along with the accelerator $(0.5 \% \mathrm{w} / \mathrm{w})$, following which the free radical initiator was added $(2 \% \mathrm{w} / \mathrm{w})$ and thoroughly mixed. The filler was added on weight basis, on parts per hundred grams of resin (phr), i. e. $2.5 \mathrm{phr}$ would refer to 2.5 grams of filler per 100 grams of unsaturated polyester resin. The composition was then deaerated to remove any entrapped air and poured in a metal mold maintained at $30^{\circ} \mathrm{C}$. The cure cycle was $30^{\circ} \mathrm{C} / 12$ hours and $100^{\circ} \mathrm{C} / 2$ hours. The composites had a thickness of $3 \mathrm{~mm} \pm 1 \%$.

The composites were machine cut into the respective shapes for testing and the edges were uniformly ground to remove imperfections which could lead to errors in the test results. Prior to testing the samples were allowed to stabilize at $50 \%$ humidity and at $30^{\circ} \mathrm{C}$ for 7 days.

\subsection{Testing of composites}

The composites were tested for their tensile, flexural and impact properties. The tensile strength and percentage elongation (Specimen Type IVB) and flexural strength (specimen dimensions $60 \times 25 \times$ $3 \mathrm{~mm}$ ) were determined according to ASTM D638 and ASTM D790 respectively on a Universal Tensile Testing Machine, Lloyd LR 50K, UK with a crosshead speed of $6 \mathrm{~mm}$ per minute for tensile and $8 \mathrm{~mm}$ per minute for the three point bending test. Charpy impact, on notched specimens of dimensions $(80 \times 10 \times 3 \mathrm{~mm}$ with a $1 \mathrm{~mm}$ notch), was carried out on an Avery Denison Impact tester using $2.7 \mathrm{~J}$ striker with a striking velocity of $3.46 \mathrm{~m} / \mathrm{s}$ in accordance with ASTM D256. Tests were carried out on five samples and the averages have been reported as the test results. 
Water and toluene absorption were studied using three $1 \times 1 \times 0.3 \mathrm{~cm}$ chips and suspending them separately for a period of 7 days in $100 \mathrm{ml}$ solvent at $30^{\circ} \mathrm{C}$. After the required amount of time the samples were removed and gently dried using a filter paper to remove water or solvent adhering to its surface. The increase in weight was then estimated and the percentage increase in water or solvent was reported.

\section{Results and discussion}

Guar gum is a very hydrophilic polymer and while most resins are hydrophobic in nature, the use of guar gum as a filler may result in a composite with increased interfacial tension. The effect of hydroxypropylation on the gaur gum would be to increase the hydrophobic nature of the polymer, while leaving the number of hydroxyl groups per pyranose unit in the polysaccharide constant, leading to increased polymer-filler interaction. This increase in the polymer-filler interaction should be reflected by an increase in the tensile and flexural strength [22] as well in other mechanical and chemical properties. The examination of the particle shape of guar gum using optical microscopy showed that the guar gum particles were highly irregular in shape as seen from Figure 1. Observation of HPG4 and HPG8 showed similar particle shapes and sizes.

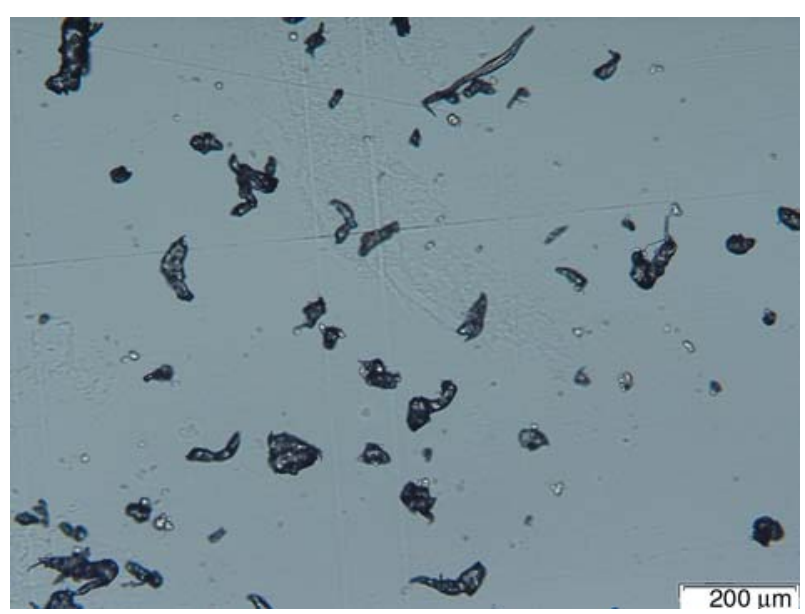

Figure 1. Image of guar gum (GG)

\subsection{Rheology}

The study of the rheology of the composition was carried out to ascertain the degree of interaction between the uncured resin and the filler. The concentration of filler taken was $2.5 \mathrm{phr}$. From Figure 2 it becomes apparent that the addition of filler

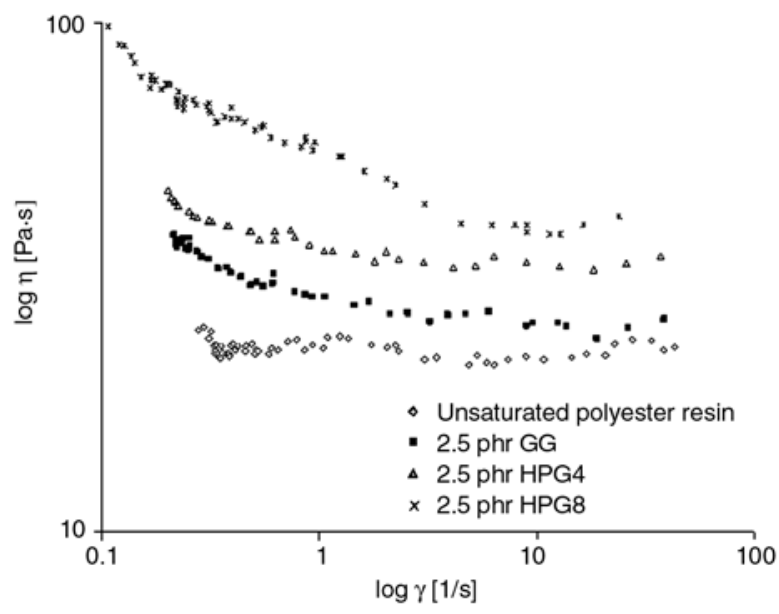

Figure 2. Variation of viscosity with shear rate

in unsaturated polyester resin results in compositions with increased viscosity, which is to be expected and has been reported in a number of studies [23]. Further, the addition of the various fillers results in compositions with varying viscosities. The viscosity of the composition increases with the degree of hydroxypropylation. Thus, HPG8 results in the most viscous composition followed by HPG4 and GG at the same concentrations of filler. The viscosity of the formulations was studied at the same concentration of filler and thus the observed increase in viscosity with degree of hydroxypropylation was due to increased interaction between the filler and polymer. An increase in the interaction between the filler and the polymer would result in an increased resistance to flow on application of shear leading to the increased viscosity. The increase in interaction can be attributed to the increased hydrophobic nature of the filler with increased hydroxypropyl content. The increase in viscosity of a polymer-filler composition through the increase in their interaction keeping other factors such as filler concentration, size, shape, etc. constant has been previously reported [24]. Although the polyester is in its uncross-linked form we assume a similar trend in the interaction between the filler and the cross-linked polyester. Further, evidence of the increased interaction will be obtained from the mechanical properties of the resultant composites.

\subsection{Solvent absorption}

Unsaturated polyester composites are known for their poor resistance to aromatic solvents, due to the use of styrene as a reactive diluent, which is 


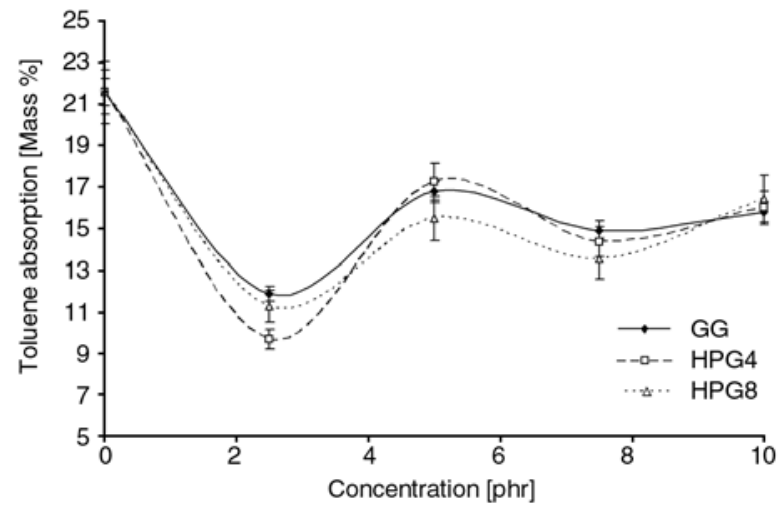

Figure 3. Toluene absorption of unsaturated polyester composites

why toluene was chosen to observe the effect of filler addition on the solvent resistance of the composites. From Figure 3 it becomes apparent that the toluene absorption reduced with the addition of filler. The filler, i. e. GG and HPGs are hydrophilic in nature and their affinity with toluene is quite low. We see that the toluene absorption of the composites do not show significant difference in the absorption behaviour with use of HPG as compared to GG. At a $2.5 \mathrm{phr}$ concentration the composites showed reduced toluene absorption which then increased on further addition and reached a steady state above 5 phr.

Considering the hydrophilic nature of the fillers the water absorption of the resultant composites becomes very important. From Figure 4 we can see that the addition of $2.5 \mathrm{phr}$ GG and HPG8 resulted in composites with reduced water absorption, which then increased with filler concentration. On the other hand the addition of HPG4 based unsaturated polyester composites did not show any significant increase in water absorption. The overall observations show that above the concentration of about $4 \mathrm{phr}$ the addition of GG as a filler resulted in

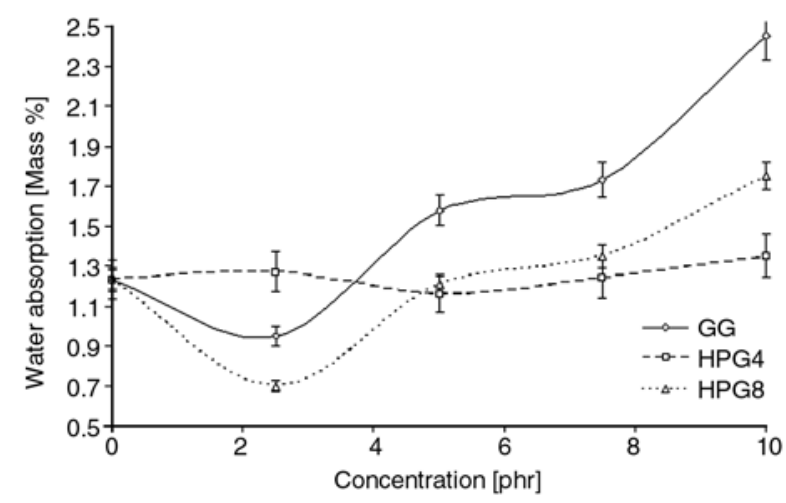

Figure 4. Variation in water absorption composites with the greatest water absorption as compared to HPGs. This can be explained by the reduction in the hydrophilic character of the substituted guar gums and also by the increased polymerfiller interaction restricting permeability of water in the composite.

\subsection{Tensile properties}

In general it was observed that there was an increase in the tensile strength of the composites containing fillers as is graphically depicted in Figure 5. Guar gum based composites also showed an increase in the tensile strength, indicating that there was some degree of interaction between the filler and the polymer matrix. The composites based on HPG4 and HPG8 showed increased tensile strength compared to that of GG based unsaturated polyester composites. HPG8 based composites showed the highest tensile strength followed by HPG4 and GG based composites. This can be explained by the increased polymer-filler interaction with increased hydrophobic nature of the filler. The optimum concentration of filler was found to be at $2.5 \mathrm{phr}$ and was found to be the same for GG and HPGs. The decrease in the tensile strength at filler concentrations above $2.5 \mathrm{phr}$ could be due to agglomerate formation which would result in the formation of stress centres in the composite contributing to initiation of catastrophic failure of the composites on the application of stress.

The percentage elongation of the composites showed an initial decrease where they showed an increase in tensile strength i.e. at $2.5 \mathrm{phr}$ followed by a maxima observed at $5 \mathrm{phr}$ as is evident from Figure 6 . Above $5 \mathrm{phr}$ the percentage elongation decreased but was still greater than that observed for unfilled unsaturated polyester in the case of

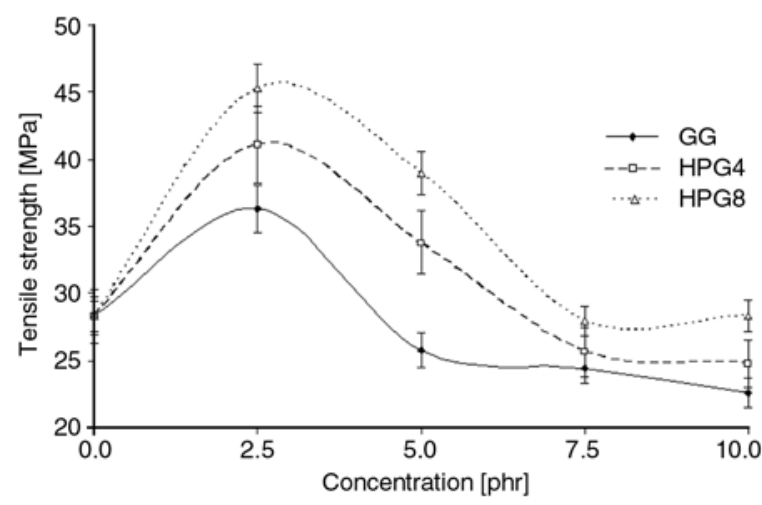

Figure 5. Variation of tensile strength with addition of filler 


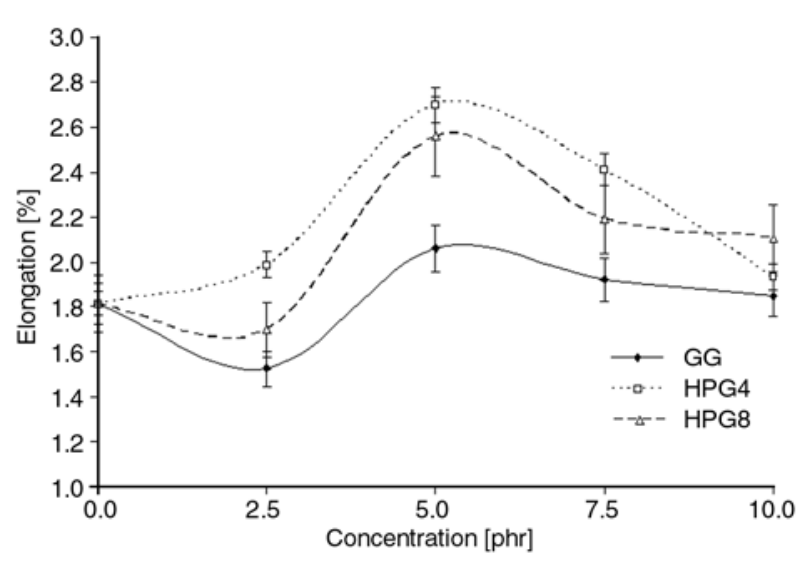

Figure 6. Variation in \% elongation

HPG based composites. GG based composites showed percentage elongation almost equivalent to that of the pure unsaturated polyester. As in the case of the tensile strength the composites based on HPG8 showed the greatest increase in the percentage elongation. The initial decrease in the percentage elongation at $2.5 \mathrm{phr}$ could be due to the reinforcing effect of the filler, which resulted in an increase in the tensile strength. At $5 \mathrm{phr}$ and beyond agglomerate formation, which resulted in a decrease in the tensile strength, could be the cause of the anomalous increase in the elongation. The elongation of the composite can also increase as a result of decreasing cross-linking density, however, if that were the case then there should have been a corresponding increase in the toluene absorption by the composites, which was not observed. Hence, a decrease in the cross-linking density as the cause of the increased elongation can be ruled out.

\subsection{Flexural properties}

The flexural strength of the composites can be seen in Figure 7 where HPGs show an increased flexural strength, with HPG8 again resulting in the composites with greatest flexural strength. HPG8 based

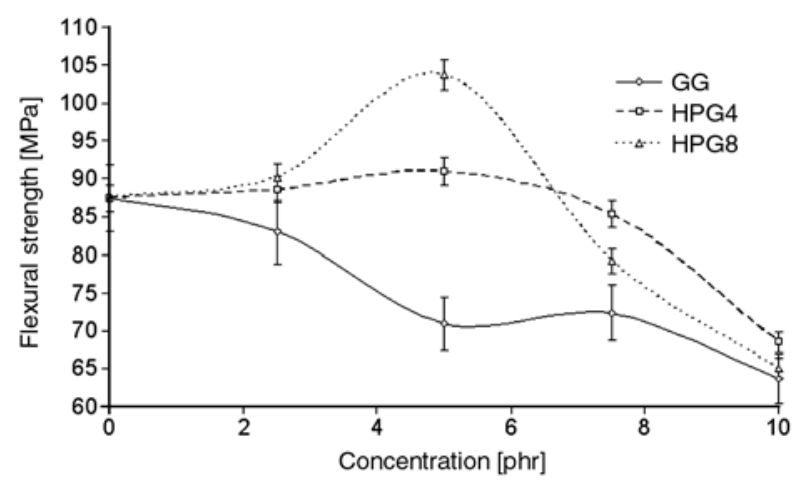

Figure 7. Variation in flexural strength composites show a maxima at $5 \mathrm{phr}$ while those based on HPG4 show an almost steady value till $7.5 \mathrm{phr}$ after which it decreases. Guar gum however, results in composites with a continuous decrease in the flexural strength with GG concentration. This trend can again be explained on the basis of increased filler-polymer interaction in the case of HPGs as compared to GG, resulting in composites with reduced internal stresses and reinforcing action of the HPG particles. The decrease in the flexural strength of the composites can be explained on the basis of agglomerate formation at higher concentrations of the filler, which was also reflected in the tensile behaviour of the composites.

\subsection{Impact properties}

Figure 8 shows the impact strength of the composites based on GG and HPG. The impact strength of the HPG based composites showed a maximum at 5 phr with HPG8 surpassing HPG4 based composites. GG resulted in composites with slightly reduced impact strength which remained almost unaffected by the increase in filler concentration. The increased impact strength of the HPG based composites can be explained by increased polymerfiller interaction as compared to GG based composites. The increased impact resistance can be explained by the formation of a tortuous fracture path which retards crack propagation. Further, increased polymer-filler interaction will decrease interfacial tension and reduce chances of crack initiation at the interface. With increasing interfacial interaction there could also be more efficient energy transfer to the filler particles which would help dissipate this energy through breakage of temporary bonds such as hydrogen bonds which are abundantly present in the polymer. All these factors

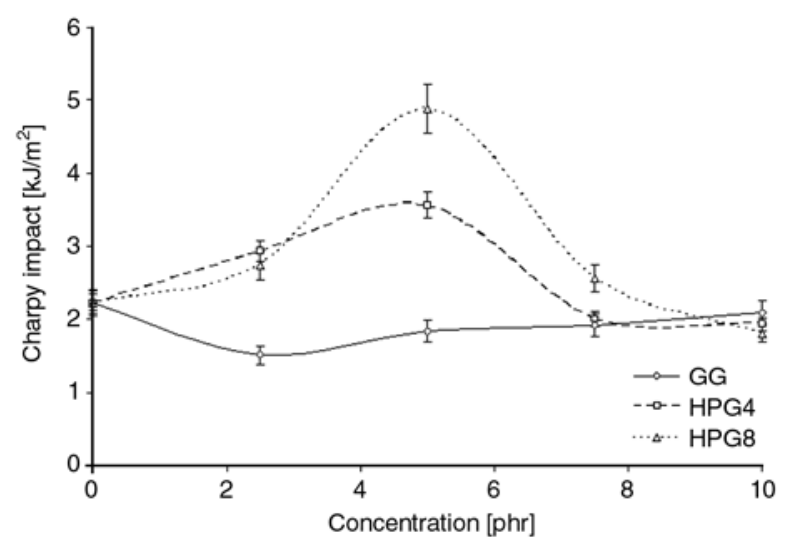

Figure 8. Variation in impact strength 
will contribute towards the increased impact resistance of the HPG based unsaturated polyester composites. At higher concentrations due to agglomerate formation, as indicated by the other mechanical properties, the impact resistance of the composites will decrease, which explains the decrease observed after $5 \mathrm{phr}$ in the impact resistance.

From the results we see that guar gum and its derivatives behave as reinforcing fillers. The hydroxypropylation of the guar gum results in increased polymer-filler interaction, both in the uncured resin as well as in the composite as indicated by the increased viscosity of the uncured formulation and improved mechanical and chemical properties of the composites. The improved performance of the hydroxypropyl guar gum fillers has been attributed to an increase in physical interaction. The use of acrylated guar gum derivatives is currently under investigation and preliminary results indicate that the use of acrylated guar gum forms an interpenetrating network. From these results other polysaccharide derivatives, such as ethers, esters and graft copolymers, of commonly available polysaccharides can also be investigated for their use as fillers. These composites can be used in applications where increased mechanical and impact properties are required by the use of an eco-friendly filler.

\section{Conclusions}

Guar gum and its hydroxypropyl derivatives were found to behave as reinforcing fillers. The increase in the degree of hydroxypropylation of guar gum increased the interaction between the polymer and polysaccharide. The toluene absorption of the composites were observed to decrease with filler addition, on the other hand the water absorption increased at filler concentrations greater than $5 \mathrm{phr}$. Composites prepared using guar gum and hydroxypropyl guar gum as fillers showed increased mechanical properties. The composites also showed increased elongation at $5 \mathrm{phr}$. On comparing the efficiency of the fillers it was observed that an increase in the degree of hydroxypropylation resulted in composites with increased mechanical properties. This opens an avenue for the use of similar polysaccharides and their derivatives as fillers in polymer composites with thermoset resins. The advantage of using such materials lies in their inherent bio-degradability, ease of ability from renewable sources and non-toxicity.

\section{References}

[1] Wientjes R. H. W., Duits M. H. G., Jongschaap R. J. J., Mellema J.: Linear rheology of guar gum solutions. Macromolecules, 33, 9594-9605 (2000).

[2] Painter T. J., Gonzalez J. J., Hemmer P. C.: The distribution of d-galactosyl groups in guaran and locustbean gum: new evidence from periodate oxidation. Carbohydrate Research, 69, 217-226 (1979).

[3] Grasdalen H., Painter T. J.: N.M.R. Studies of composition and sequence in legume-seed Galactomannans. Carbohydrate Research, 81, 59-66 (1980).

[4] Sostar S., Schneider R.: A study of fabric stiffness with guar gum in reactive printing. Dyes and Pigments, 41, 167-175 (1999).

[5] Schneider R., Sostar-Turk S.: Good quality printing with reactive dyes using guar gum and biodegradable additives. Dyes and Pigments, 57, 7-14 (2003).

[6] Funami T., Kataoka Y., Omoto T., Yasunori G., Asai I., Nishinari K.: Food hydrocolloids control the gelatinization and retrogradation behavior of starch. $2 \mathrm{a}$. Functions of guar gums with different molecular weights on the gelatinization behavior of corn starch. Food Hydrocolloids, 19, 15-24 (2005).

[7] Maurin V., Beauquey B.: Composition for washing keratin materials, based on a detergent surfactant, a cationic galactomannan gum and an acrylic terpolymer. U. S. Patent 6383993, USA (2002).

[8] Soppimath K. S., Kulkarni A. R., Aminabhavi T. M.: Chemically modified polyacrylamide-g-guar gumbased crosslinked anionic microgels as $\mathrm{pH}$-sensitive drug delivery systems: preparation and characterization. Journal of Controlled Release, 75, 331-345 (2001).

[9] Moorhouse R., Matthews L. E.: Aqueous based zirconium (IV) crosslinked guar fracturing fluid and a method of making and use therefore. U. S. Patent 6737386, USA (2004).

[10] Mothe C. G., Correia D. Z., de Franca F. P., Riga A. T.: Thermal and rheological study of polysaccharides for enhanced oil recovery. Journal of Thermal Analysis and Calorimetry, 85, 31-36 (2006).

[11] Ayman M. A., Abdel-Raouf M. E., Elsaeed S. M., Abdel-Azim A. A.: Curable resins based on recycled poly(ethylene terephthalate) for coating applications. Progress in Organic Coatings, 55, 50-59 (2006).

[12] Lu W., Lin H., Wu D., Chen G.: Unsaturated polyester resin/graphite nanosheet conducting composites with a low percolation threshold. Polymer, 47, 4440-4444 (2006).

[13] Burgueno R., Quagliata M. J., Mohanty A. K., Mehta G., Drzal L. T., Misra M.: Load-bearing natural fiber composite cellular beams and panels. Composites Part A- Applied Science and Manufacturing, 35, 645-656 (2004).

[14] Pietschmann N., Stengel K., Hoesselbarth B.: Investigations into vinylogic addition reactions of modified polyester resins. Progress in Organic Coatings, 36, 64-69 (1999). 
[15] Martuscelli E., Musto P., Ragosta G., Scarinizi G.: A polymer network of unsaturated polyester and bismaleimide resins: 1 . Kinetics, mechanism and molecular structure. Polymer, 37, 4025-4032 (1996).

[16] Banu P., Radhakrishnan G.: Unsaturated poly(esterimide)s from hydroxy-terminated polybutadiene, dianhydride and diisocyanate. European Polymer Journal, 40, 1887-1894 (2004).

[17] Vargha V., Truter P.: Biodegradable polymers by reactive blending trans-esterification of thermoplastic starch with poly(vinyl acetate) and poly(vinyl acetateco-butyl acrylate). European Polymer Journal, 41, 715-726 (2005).

[18] Yang H. S., Wolcott M. P., Kim H. S., Kim H. J.: Thermal properties of lignocellulosic filler-thermoplastic polymer bio-composites. Journal of Thermal Analysis and Calorimetry, 82, 157-160 (2005).

[19] Caraschi J. C., Leao A. L.: Woodflour as reinforcement of polypropylene. Material Research, 5, 405409 (2002).
[20] Kumar H., Siddaramaiaha Somashekar R., Ranganathaiah C.: Biodegradation and characterization of guar gum filled polyurethane/polyacrylonitrile semiinterpenetrating polymer network. in 'MACRO 2006 - $9^{\text {th }}$ National conference of polymers for advanced technologies' NCL Pune, India, 1-2 Dec (2006).

[21] Huang Y., Xiao C.: Miscibility and mechanical properties of quaternized polysulfone/benzoyl guar gum blends. Polymer, 48, 371-381 (2007).

[22] Sen S., Nugay N.: Tuning of final performances of unsaturated polyester composites with inorganic microsphere/platelet hybrid reinforcers. European Polymer Journal, 37, 2047-2053 (2001).

[23] Joo Y. L., Lee Y. D., Kwack T. H., Min T. I.: Rheology and processing of polycarbonate/carbonate/carbon black composites. in 'Antec '96. Conference Proceedings', Indianapolis, USA, Vol. I., 64-68 (1996).

[24] Clarke J., Freakley P. K.: Reduction in viscosity of an SBR compound caused by mastication and disagglomeration during mixing. Rubber Chemistry and Technology, 67, 700-715 (1994). 